\title{
DEPLOYMENT OF ELECTRONIC MEDICAL PRESCRIPTIONS FOR AMBULATORY CARE IN BELGIUM TRACING AND ELIMINATING TECHNICAL ERRORS
}

\author{
Marc Nyssen ${ }^{1}$ and Yelina Piedra Salomón ${ }^{2}$ \\ ${ }^{I}$ Independent Author, JB. Vandercammenstraat 25, Brussels, Belgium \\ ${ }^{2}$ University of Havana, Havana, Cuba
}

\begin{abstract}
Electronic prescriptions in ambulatory care are considered to be the second most important e-health goal, after the electronic medical record in many countries. Belgium is no exception: electronic prescription are part of the successive national e-health plans since more than 10 years. This paper reviews the scientific production on the topic of electronic prescriptions (via the Dimensions database). In the second part, we then explains the fundamental principles that were adopted and faithfully realized, the main functional characteristics and the technical realization and deployment of the ambulatory electronic prescription system "Recip-e"in Belgium. Then we highlight the evolution of the main parameters and technical problems that were encountered during the roll-out phase of the Recip-e project and the remaining challenges.
\end{abstract}

\section{KEYWORDS}

Electronic Prescription, e-Prescription, Ambulatory Care, e-Health, Deployment

\section{INTRODUCTION}

Since 2005, the World Health Organization [WHO 2005] encourages its member states to define the national e-health plans and even provides a "toolkit" to facilitate these exercises. Many countries have elaborated such plans and usually, electronic medical records and electronic prescriptions (intra-muros as well as ambulatory) are cornerstones of such plans. In the EU, the EPSOS project developed the tools for trans-border exchange of summary records and electronic prescriptions, this was evaluated in the field [Moharra 2015] and will most probably be followed by an effective deployment during the following years.

In this paper we first make a literature survey, on the subject of the electronic prescriptions in ambulatory care, to show the importance of the related scientific production, the evolution in time of relevant scientific publications and the international relationships that link these publications to one another.

The second part of the paper explains the architecture of the ambulatory electronic prescription system in Belgium and shows the results of the deployment since 2011, in three different stages.

The purpose of this paper is to give insight in the field of ambulatory electronic prescription in general and to focus on a specific country-wide implementation that is now mature enough so that lessons can be learned which will help others in optimizing their implementations. These deployments will have to take into account the common requirements that underly electronic prescribing but will also adapt to the specific characteristics of the health care system of each country.

\section{ELECTRONIC AMBULATORY PRESCRIPTIONS}

After making a comprehensive literature search, we will focus on the implementation and deployment in Belgium. 


\subsection{Literature Research}

For the systematic collection of scientific production on the subject, we used the Dimensions database ${ }^{1}$. This is a linked research knowledge system that offers novel approaches to discover relevant publications and access to research. Developed by Digital Science in collaboration with over 100 leading research organizations around the world, Dimensions encompasses grants, publications, citations, alternative metrics, clinical trials, patents and policy documents to deliver a platform that enables users to find and access the most relevant information faster, analyze the academic and broader outcomes of research, and gather insights to inform future strategies (Dimensions, 2019).

By searching "electronic prescription" OR "electronic prescriptions" OR "e-prescription", a total of 582 documents were collected. We found 428 articles, 68 chapters, 50 books, 33 proceedings and 3 monographs. A total of 194 patents and 34 policy documents were also part of the sample. We limited further work to articles, books and proceedings because an organizational subscription is requested by the database to access the other types, which seemed also less relevant to us for this study. Microsoft Excel and Tableau Public 2018.3 were used to generate the frequency tables. For the bibliometric mapping of titles, co-word analysis and countries collaboration networks the data was exported to a .cvs file. To construct and visualize bibliometric networks we used VoSviewer 1.6.10.

The most productive authors on the topic, based on the number of published articles are Michelle Anne Chui (13) from University of Wisconsin-Madison, United States; Olufunmilola K Odukoya (10) from University of Pittsburgh, United States and Johanna Timonen (10) from University of Eastern Finland, Finland. The first two authors form an invisible group because they collaborate in publishing. Their most relevant researches according database quotation counts are focused on exploring the consequences and contributing factors of e-prescribing errors in community pharmacies [Odukoya, Stone \& Chui, 2014], this article obtained 30 quotations. The second one obtained 26 quotations and focuses in exploring pharmacy staff perceptions of the strengths and weaknesses of electronic prescribing (e-prescribing) design in retail pharmacies using the socio-technical systems framework [Odukoya \& Stone, 2011].

The first contribution (with 6 quotations) is a semistructured interview among primary health care physicians in Finland to find out about how medication and the usability of electronic prescribing is perceived by physicians [Kauppinen, Ahonen, Mäntyselkä \& Timonen, 2017]. The second [Kauppinen, Ahonen \& Timonen, 2017] and the third [Lämsä et al, 2017] most relevant works of Timonen obtained 5 quotations each. These publications mainly focus on the impact of electronic prescriptions on medication safety in Finnish community pharmacies, using a survey of pharmacists and in the pharmacy customer's experiences with My Kanta, the national onl-ine service for viewing electronic prescriptions in Finland.

The most cited document of the whole sample is authored by Fisher et al (2010). This article is focused on evaluating primary care non-adherence in community-based practices and identifies predictors of non-adherence. The authors concludes that many e-prescriptions were not handed-in to pharmacies and previous studies of medication non-adherence failed to capture these prescriptions. Efforts to increase primary adherence could dramatically improve the effectiveness of medication therapy.

Interventions that target specific medication classes may be most effective (citation-wise). According to the database, this publication in Journal of General Internal Medicine has been cited 260 times. 32\% of its citations have been received in the past two years, which is higher than you might expect, suggesting that it is currently receiving a lot of interest. Compared to other publications in the same field, this publication is scores very high regarding citations as it has received approximately 28 times more citations than average. This research output has an Altmetric Attention Score of 409, as was calculated when the research output was last mentioned on 16 October 2017. This is the Dimension's high-level measure of the quality and quantity of on-line attention that it has received. It's in the top 5\% of all research outputs ever tracked by Altmetric ${ }^{2}$. This publication was found relevant by 18 Dimension's categories ${ }^{3}$ according to different research fields.

\footnotetext{
${ }^{1}$ For more information about the product visit https://dimensions.ai.

2 https://dimensions.altmetric.com/details/1373293/citations

3 Public Health and Health Services, Information Systems, Clinical Sciences, Policy and Administration, Data Format, Applied Economics, Artificial Intelligence and Image Processing, Computer Software, Law, Business and Management, Psychology, Cardiorespiratory Medicine and Hematology, Pharmacology and Pharmaceutical Sciences, Sociology, Computation Theory and Mathematics, Specialist Studies in Education, Communications Technologies, Nursing, Political Science, Physical Chemistry (incl. Structural), Veterinary Sciences, Curriculum and Pedagogy, Banking, Finance and Investment, Linguistics, Philosophy
} 
In the period between 1989 and 2019, the scientific production collected was as depicted in Figure 1:

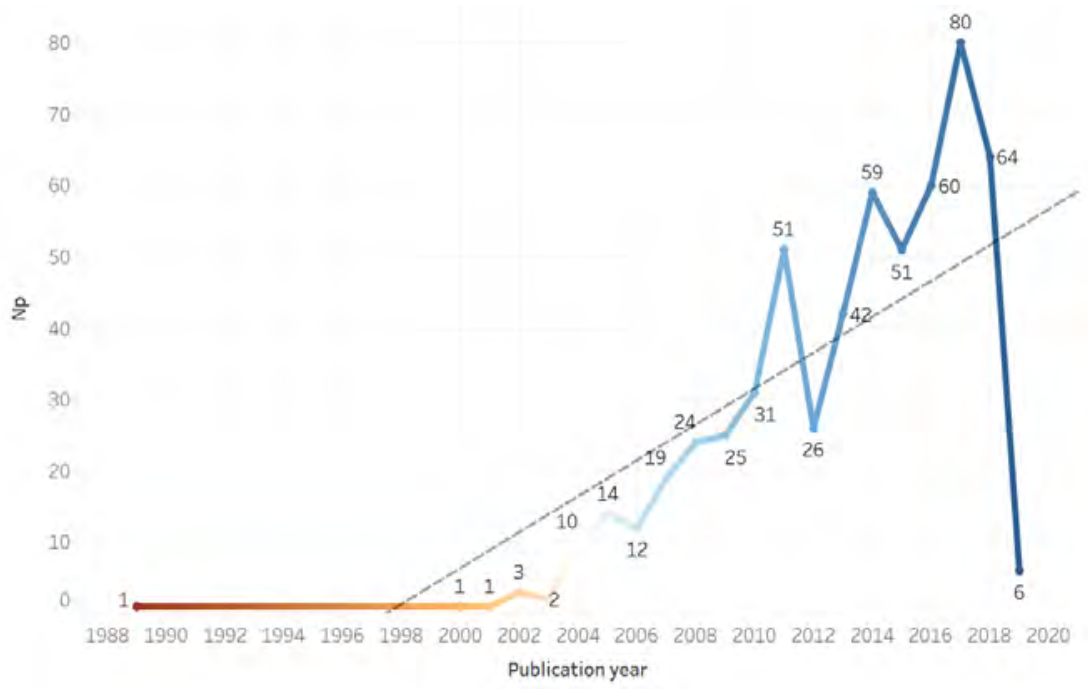

Figure 1. Chronological distribution of electronic-prescriptions scientific production in Dimensions

As shown, in general, there is a growing trend line mainly starting in 2005 , indeed the period in which electronic prescription systems became operational in several regions of the world. Also in Belgium the first designs and proposals for a national electronic prescription system in ambulatory care date from that period.

\subsection{Deployment of Electronic Prescriptions in Belgium}

\subsubsection{General Principles}

Ambulatory prescriptions in Belgium originate from (independent) physicians, dentists, midwives and hospitals (upon release of patients), while the medication delivery is performed in independent pharmacies (4840 country-wide). Patients can freely choose any pharmacy. Prescribers make use of electronic medical record systems, which all have prescription modules (either in private practice, in polyclinics, hospitals), provided by 25 different software vendors. All pharmacies have pharmacy information systems, provided by 8 vendors.

It was decided to make maximal use of the national "eHealth-platform", guaranteeing safe and secured data transmission and a user-and-access management system comprising all health care workers. Patients are identified by their national identifier (not only Belgians, but also given to all inhabitants residing at least three months in the country).

\subsubsection{Implementation Methodology}

In order to keep the prescriptions, submitted by prescribers, available to the pharmacist, freely chosen by the patient, we developed a secure database application: "Recip-e engine". Enabled and authentified prescribers submit prescriptions uniquely identified prescriptions in KMEHR-XML format to this database. The unique identifiers (Recip-e Identifier or RID) are attributed by the central service upon creation.

The pharmacists, chosen by the patient can deliver the medication and thereby realize a status change in the server, prohibiting thereby multiple deliveries of a prescription; finally archiving by the pharmacist enables the Recip-e server to delete the prescription from the database, thereby releasing space. 


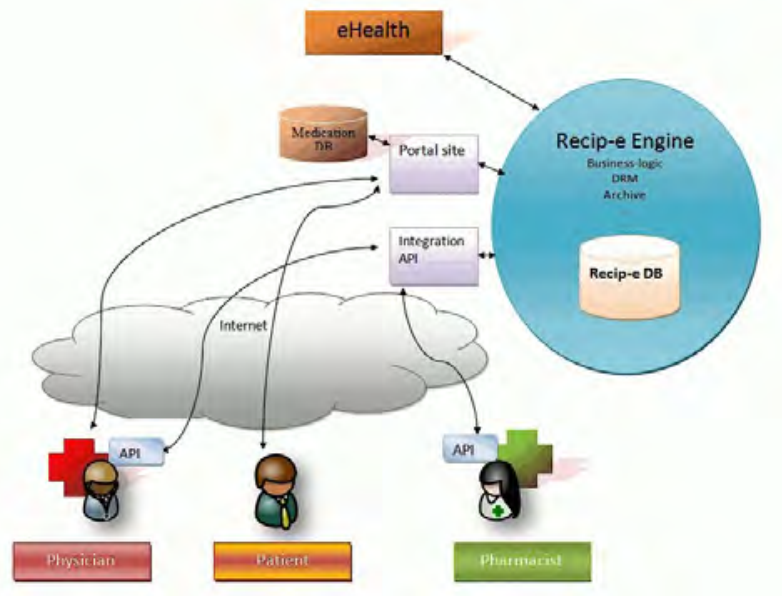

Figure 2. General Recip-e Architecture

The Recip-e system was realized by a not for profit organization: Recip-e, managed by representatives of all medical professions. The financing came from the Belgian Federal Social Security organization RIZIV/INAMI.

Three implementations were realized: "test", "acceptance" and "production". The "test" environment allows developers of the central software to develop and test new server releases. Once OK, these are transferred to "acceptance" which is a complete eHealth-platform environment, allowing third parties (software vendors for prescribers and pharmacies) to test their end-user applications by calling services such as Recip-e, but also others: sharing of medical records, requesting information on patient's insurance statuses and sending in or consulting summary records, as typical examples. The "production" environment is the real thing: severely controlled and updated only a few times per year with enforcement of strict regulations regarding access and identity controls.

Triple encryption end-to-end assures security, but also limits the controls than can be provided centrally. We can just rely on "header information" to assure good technical functioning; content-wise we are blind: the prescriber and the executors are supposed to use the same XML formats, medication databases and codes as well as structured posologies in their prescription messages.

\subsubsection{Deployment Time-Line}

The deployment in production (since 2011) was accompanied by regulations by the state: first phase 2011-2016 paper prescriptions simultaneous with electronic prescriptions identified by a RID (unique identifier per prescription) bar-code on top of the paper print-out; second phase: 2017-2020 electronic prescriptions with printout of a "proof of electronic prescription" containing the RID and medication print-out but without any legal value of the paper, just assisting recovery of the electronic prescription (usually via the bar-code readers at the pharmacies); third phase: starting January 2020 - obligatory usage of electronic prescriptions, possibly without printout of a "proof of electronic prescription" and recovery at the pharmacy via the identity of the patient.

Deployment was progressive, as presented in the graph below, showing the monthly figures of three key indicators: the number of prescriptions received from prescribers (top blue line), the number of prescriptions, delivered by pharmacists (center red line) and the number of prescriptions archived by pharmacists (lower green line). 


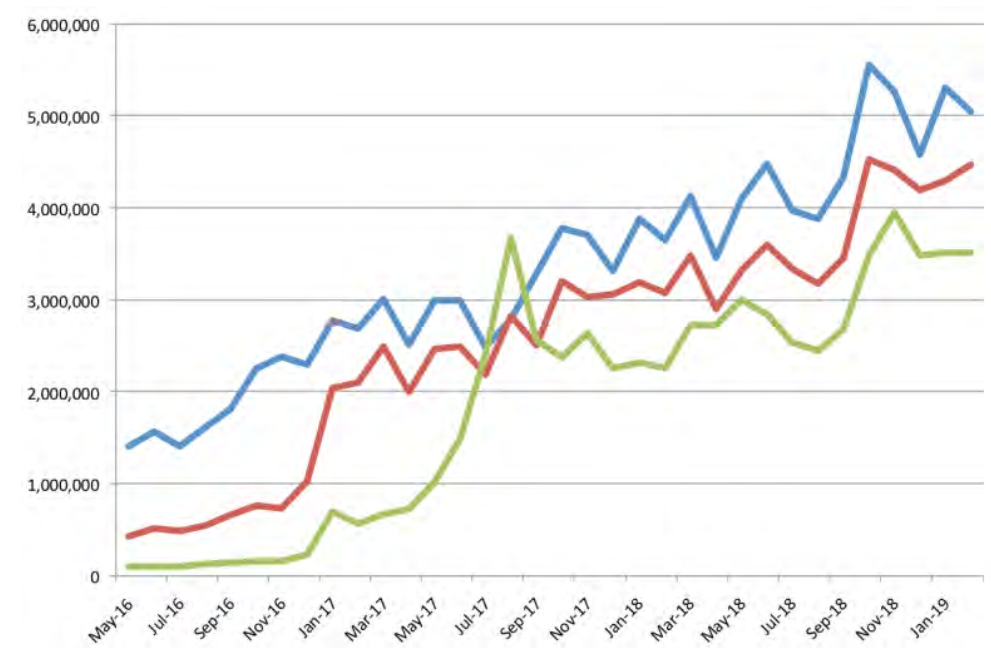

Figure 3. Key Deployment Indicators (May 2016 - February 2019)

In January 2017, a sharp rise in volume was due to the introduction of the "proof of electronic prescription" (with no legal value) that needs to be accompanied by the electronic prescription in the pharmacy, as the only legal document if no paper based prescription is present.

The graph shows that at the beginning of 2019, we reached the 5 million incoming electronic prescriptions per month milestone. Top days are Mondays, with just over 300.000 incoming prescriptions.

\subsubsection{State Diagram}

The Recip-e system was formally described as a "state machine", defining the states in which every electronic prescription is at any moment in time. State modifications are activated by the corresponding webservice calls, emanating directly from the systems of the users (prescribers and pharmacists). When a prescriber creates a new prescription on his/her electronic medical record system, the electronic version of this prescription is then transmitted to the Recip-e server with the Create_Prescription webservice. Each new prescription, identified by its unique Recip-e ID obtains the status: "Not Delivered"; meaning that any pharmacy can now pick it up via a "Get Prescription" webservice call, emanating directly from the pharmacy.

This is illustrated in Figure 3. Suppose a pharmacy gets the prescription by activating the webservice "Get_Prescription RID", at this point the status is changed into "In Process", meaning that no other pharmacy can obtain it: the prescription is "locked" at the first pharmacy, where at this point any of three transition possibilities can take place: either the pharmacist delivers the medication, present in the prescription, his system must then communicate "Mark_as_delivered" and the status of the prescription moves to: "Delivered"; otherwise, the prescription is "handed back to the patient", no delivery takes place and the patient can go to any other pharmacy or come back later: this is indicated by the "Mark_as undelivered" webservice and results again in the status: "Not Delivered"; finally, the pharmacist can "revoke" the prescription, as it is no longer useful by "Mark_as_revoked": the status becomes "Revoked": the prescription can no longer be used, its contents can be deleted.

After delivery, the pharmacist will make sure the prescriptions which he delivered are properly archived, either by himself or by an external instance (tarification/archiving service), this must be confirmed to the Recip-e server via a "Mark_as_archived" webservice call, resulting in the status: "Archived".

On daily basis, the Recip-e server deletes the prescription with status "Archived" from the database, to recover storage space.

Webservices play an eminent role in the whole e-health environment, as they are well defined and allow strict syntax and user access controls. 


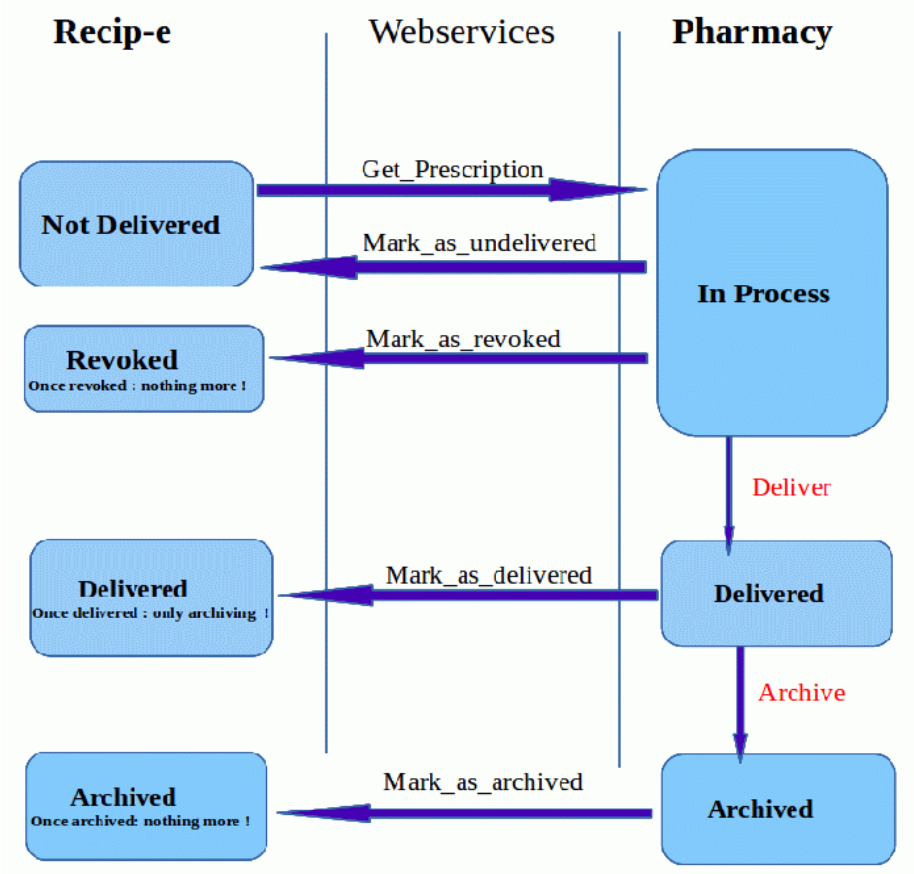

Figure 4. State Diagram of a Prescription and Corresponding Web-Service Calls

To make the system consistent, third party software at prescribres' locations and pharmacies should keep trace of the statuses of all the prescriptions they manage, following-up on the pharmacist's actions immediately so that there is coherence between the status in the Recip-e database and the effective status "in the field".

Note that prescribers can also revoke prescriptions (for example during the consultation when they realize they made an error) by performing Mark_as_revoked, but of course only for the prescriptions they had made.

Patients have access to the system via the "MyHealth" national portal: they can list, visualize the contents of their prescriptions and delete those they don't need anymore by issuing revoke requests.

Currently, about 130 patients consult their prescriptions every day, but only 3 prescriptions are revoked by them.

\subsubsection{Problem Follow-up and Remedies}

The Recip-e system has an audit trail with associated web interface, allowing to follow up on every individual electronic prescription, perform searches in time windows (up to the minute), indicate conditions on prescriber, executor and other conditions such as error codes.

This audit trail is used for follow-up purposes, to discover erroneous operations and to pinpoint their origins.

Table 1 shows the observed error rates, associated with the different end-user operations, at two Mondays, separated by 1 year: March $5^{\text {th }} 2018$ and March $6^{\text {th }} 2019$.

On March $5^{\text {th }} 2018,9777$ prescribers $(8630$ general practitioners, 431 independent specialists, 961 dentists and 25 hospitals sent in 207804 prescriptions.

On March $4^{\text {th }} 2019,10409$ prescribers (mainly GP's: 8517, but also 714 independent specialists, 1055 dentists, 1 midwife and 122 hospitals) sent in 246027 prescriptions.

The increase in total users is mainly due to the hospitals (there are a total of 132 hospitals in the country). 
Table 1. Error Rates (Comparison between March 2018 and March 2019)

\begin{tabular}{|l|c|c|c|c|c|}
\hline \multicolumn{1}{|c|}{ Function } & Prescribers & Pharmacies & Patients & $\begin{array}{c}\text { Error rates } \\
\mathbf{5} \text { March 2018 }\end{array}$ & $\begin{array}{c}\text { Error rate } \\
\mathbf{4} \text { March 2019 }\end{array}$ \\
\hline Create prescription & $*$ & & & $0.27 \%$ & $0.011 \%$ \\
\hline Revoke (delete) prescription & $*$ & $*$ & $*$ & $2.6 \%$ & $6.3 \%$ \\
\hline List open prescriptions & $*$ & & $*$ & 0 & 0 \\
\hline Get prescription & $*$ & $*$ & $*$ & $1.0 \%$ & $2.0 \%$ \\
\hline Mark as delivered & & $*$ & & $.40 \%$ & $1.0 \%$ \\
\hline Mark as undelivered & & $*$ & & $9.4 \%$ & $0.98 \%$ \\
\hline Archive prescription & & $*$ & & $1.6 \%$ & $0 \%$ \\
\hline Announce prescription & $*$ & & & $0 \%$ & $0 \%$ \\
\hline Create feedback message & & $*$ & & $0 \%$ & $0 \%$ \\
\hline List feedback message & $*$ & & & $0 \%$ & 0 \\
\hline
\end{tabular}

\section{Technical Errors due to Prescribers}

At the input side, we observe tree types of technical errors, leading to non-admission of an electronic prescription into the system:

- identity problems: the identity of the physician who started the session (SAML token, which we consider as the signature, validated by the prescriber's electronic certificate and electronic signature with his e-ID) is not the same as the identity of the prescriber in the header of the prescription;

- $\quad$ wrong prescription format (name or header badly formatted by the prescriber software)

- technical error: time-stamping service not available or other server errors

Each of these accounts for about equal portions of the 2018 measurement: $0.09 \%$ of incoming prescriptions.

Improving the bandwidth of the main bus, capacity of the time-stamping server at the eHealth-Platform and follow-up towards the vendors of prescribing softwares, virtually eliminated the last two reasons for technical errors; currently only the first (prescriber taking over a session of a colleague) one remains.

We try to eliminate these errors by strongly recommending vendors of prescribing packages to re-check the correspondence of the identity of the prescriber versus the one in the current SAML token, before sending in a prescription and thus avoiding useless transmissions, error messages and possible user frustration.

Prescribers can revoke prescriptions they have made, surprisingly, the absolute numbers of successful revokes by prescribers (from 771 to 1488 ) have almost doubled and the error rate for this operation went up from $4.4 \%$ to $6.3 \%$.

\section{Technical Errors due to Pharmacists}

Here, the error rates are higher: the main origin seems to be the difficulty pharmacy packages have to keep track of the status of each Recip-e prescription that is handled in the pharmacy (in particular when pharmacies operate via multiple terminals) and in the interpretation of error messages.

The rather high rate of errors with the "get-Prescription" operation is due to two causes: re-doing the operation in the same pharmacy, after delivery took place and repeating the operation, not taking into account the previous error messages that were sent by the server.

Also: here we observe a few cases of "fraud attempts" by patients who try to obtain multiple deliveries, based on a single prescription.

Thanks to insisting on "good practices", the error rates regarding archiving gave gone down significantly and we hope that in 2019, the number of "archived" prescriptions will effectively equal the number of "delivered" ones. 


\section{CONCLUSION}

The literature exploration learns us that the scientific production on the topic of electronic prescriptions is still growing, that international cooperations exist and that the main topics are situated in the fields of adoption and error tracing.

Our experience with the roll-out of a large scale electronic prescription system learns that it is possible to operate with many vendors, provided that clear specifications are given and that the system's characteristics are close to the daily practice of the health care professionals who have to use it.

A comprehensive and informative audit trial system is essential in tracing technical errors and subsequent actions can be made very efficient, provided not encrypted header information of the messages contains the source (health care worker) and the tools that are used (program vendor, program name and version that is in use by the prescriber) to generate the prescription.

Precise feedback to software vendors must be given by the central system, as end-users are not capable of discerning where problems exactly originate.

\section{ACKNOWLEDGEMENT}

Thanks to the Belgian RIZIV-INAMI, sponsor of the Recip-e system; to D. Broeckx for formatting Figure 3.

\section{REFERENCES}

Fischer M A et al, 2010, "Primary Medication Non-Adherence: Analysis of 195,930 Electronic Prescriptions", Journal of General Internal Medicine, 195 p 930.

Kauppinen $\mathrm{H}$ et al, 2017, "Medication safety and the usability of electronic prescribing as perceived by physicians - A semistructured interview among primary health care physicians in Finland", Journal of Evaluation in Clinical Practice, 23(6), 1187-1194, 2017.

Kauppinen $\mathrm{H}$ et al, 2017, "The impact of electronic prescriptions on medication safety in Finnish community pharmacies: A survey of pharmacists", 1 Journal of Medical Informatics.

Lämsä E et al, 2017, "Pharmacy customers' experiences with the national on-line service for viewing electronic prescriptions in Finland", International Journal of Medical Informatics.

Moharra, $\mathrm{M}$ et al, 2015, "Implementation of a cross-border health service: physician and pharmacists' opinions from the epSOS project”, Fam. Pract, 2015, Oct, 32(5) pp. 564-567.

Odukoya $\mathrm{O}$ et al, 2014, "E-prescribing errors in community pharmacies: Exploring consequences and contributing factors", International Journal of Medical Informatics, 83(6), 427-437.

Odukoya O, Chui M A, 2012, "Retail pharmacy staff perceptions of design strengths and weaknesses of electronic prescribing", Journal of the American Medical Informatics Association, 19(6), 1059-1065.

Papshev D et al, 2001, "Electronic Prescribing in Ambulatory Practice: Promises, Pitfalls, and Potential Solutions", Am. J. Manag. Care, Jul 7 (7), pp. 725-736.

Theilgaard J, 1997, "Message Implementation Guide for Prescription", version 1.0, 21.03 .97 for the CoCo project, on behalf of Medcom, Denmark. Zipped file (120 K) of a Word-document of 117 pages.

WHO, 2005, "World Health Assembly Resolution 58.28" and "ITU World Telecomunication Development Conference Resolution 65 (2010)". 